\title{
Reduction of Leakage from Insertion Site During Continuous Femoral Nerve Block with Catheter- Through-Needle Versus Catheter-Over-Needle Technique for Postoperative Analgesia After Total Knee Arthroplasty: A Randomized Controlled Trial
}

\section{Yoshiyasu Hattammaru}

Jikei University School of Medicine

Yasushi Mio ( $\nabla$ mio@jikei.ac.jp )

Jikei University School of Medicine

\section{Tomasz Hascilowicz}

Jikei University School of Medicine

Isao Utsumi

Jikei University School of Medicine

Yuichi Murakami

Jikei University School of Medicine

Sachiko Omi

Jikei University School of Medicine

\section{Research Article}

Keywords: continuous femoral nerve block, catheter-over-needle, leakage from catheter insertion site, total knee arthroplasty

Posted Date: September 27th, 2021

DOI: https://doi.org/10.21203/rs.3.rs-728849/v1

License: (c) (i) This work is licensed under a Creative Commons Attribution 4.0 International License.

Read Full License

Version of Record: A version of this preprint was published at BMC Anesthesiology on January 5th, 2022. See the published version at https://doi.org/10.1186/s12871-021-01554-9. 


\section{Abstract}

Background: Continuous femoral nerve block (CFNB) is a common procedure used for postoperative analgesia in total knee arthroplasty. Continuous nerve block using a conventional needle (catheterthrough-needle/CTN) is complicated by leakage of the anesthetic from the catheter insertion site. A new type of needle (catheter-over-needle/ CON) is now available, which is believed to reduce leakage as the diameter of the catheter is larger than that of the needle. The purpose of this study was to compare leakage from the catheter insertion site during CFNB when using CTN and CON for postoperative analgesia after total knee arthroplasty (TKA).

Methods: This prospective, randomized, single-blinded controlled study included 60 patients who were scheduled for TKA at our facility between May 2016 and November 2017. Patients were randomly allocated to the CTN and CON groups. All patients in the two groups received CFNB and sciatic nerve block for postoperative analgesia. The administration of $0.16 \%$ levobupivacaine mixed with $6 \mathrm{mg}$ of indigo carmine (a dye added to easily identify leakage) was started at $6 \mathrm{ml} / \mathrm{h}$ at the end of surgery. The primary outcome was the incidence of leakage from the catheter insertion site. We further investigated the degree of leakage, the incidence of catheter migration, pain scores using the numerical rating scale at 48 hours postoperatively, and the number of days until the operated knee could be flexed 120 degrees postoperatively in both groups.

Results: The CON group had a significantly lower incidence of leakage from the catheter insertion site. There were no significant differences in other measurement outcomes.

Conclusions: Use of CON reduces the incidence of leakage from the catheter insertion site during CFNB in the use of postoperative analgesia for total knee arthroplasty. Future research is needed to determine additional benefits of using CON related to decreased leakage.

Trial registration: The study was registered in the University Hospital Medical Information Network (UMIN) Clinical Trials Registry (UMIN000021537), prospectively registered on 18 March 2016.

\section{Background}

Epidural anesthesia has a greater analgesic effect than systemic opioid administration or intravenous patient-controlled analgesia in postoperative analgesia and is superior in terms of the quality of analgesia [1]. Thus, epidural anesthesia is widely used for postoperative analgesia. Lower extremity surgeries such as hip and knee arthroplasty are classified as high-risk groups for deep vein thrombosis during the perioperative period, and anticoagulation therapy is commonly used postoperatively. Because the use of anticoagulants may increase the incidence of spinal (epidural) hematoma [2], epidural anesthesia tends to be avoided in patients who undergo surgeries with high-risk of deep venous thrombosis. 
In recent years, ultrasound-guided nerve blocks have partly replaced epidural anesthesia, and catheter placement as a continuous peripheral nerve block has been used in invasive surgeries. However, a continuous peripheral nerve block performed with a conventional catheter-through-needle (CTN) is associated with leakage of anesthetic from the catheter insertion site, which may lead to catheter malposition and attenuation of analgesic effects [3-5]. Catheter-over-needle (CON), a new needle for continuous peripheral nerve block has been newly made available. The advantage of CON is considered to be the lower level of leakage from the catheter insertion site compared with CTN because the diameter of the catheter is larger than that of the needle. Although several randomized clinical trials have been conducted on CTN versus CON, there have been very few trials comparing the efficacy and safety of CTN and $\operatorname{CON}[6-9]$.

This study investigated whether there was a difference in leakage from the catheter insertion site of the continuous femoral nerve block (CFNB) between CTN and CON when used for total knee arthroplasty (TKA).

\section{Methods}

\section{Data Collection}

Patients aged 20-80 years who were scheduled for monolateral TKA from May 11, 2016 to November 8, 2017 were included in this trial. The exclusion criteria included re-operation of TKA, local anesthetic allergy, infection at the insertion site, American Society of Anesthesia physical status $>$ III, daily steroid use (prednisolone $>20 \mathrm{mg} /$ day), and inappropriate entry into the study based on the judgment of the investigator. Sixty consecutive TKA patients, from whom informed consent was obtained and who fulfilled the inclusion criteria were enrolled.

Sixty patients were randomized into two groups using Microsoft Excel (Redmond, WA, USA): the CTN and CON groups. Contiplex (B. Braun, Meisungen, Germany) and Contiplex C (B. Braun, Meisungen, Germany) were used in this study in the CTN and CON groups, respectively. The randomized table was kept by an independent researcher (Y Murakami) who was not involved in anesthesia procedures or outcome assessments.

\section{Anesthetic Procedure}

All nerve blocks were performed by $\mathrm{YH}$ and SO, who have substantial experience in performing ultrasound-guided nerve blocks. The Edge2 ultrasound system linear probe (HFL50x, 6-15 MHz) (Sonosite, Bothell, USA) and Stimplex HNS12 nerve stimulator system (B. Braun, Meisungen, Germany) were used. In each case, postoperative follow-up and evaluation were performed by the same anesthesiologist who conducted the nerve blocks.

Noninvasive blood pressure, electrocardiogram, heart rate, and pulse oxygen saturation were monitored before and during the femoral nerve block procedure. The femoral nerve was identified in the inguinal 
region with a transverse cross-sectional view under ultrasound guidance. The needle tip was advanced to the dorsal side of the femoral nerve near the femoral artery, and the tip position was confirmed after administration of saline. After placement of the catheter, a local anesthetic $(0.25 \%$ ropivacaine, $10 \mathrm{ml})$ was administered through the catheter. A sciatic nerve block was then performed through the popliteal approach. General or spinal anesthesia were selected as intraoperative anesthesia. At the end of surgery, $10 \mathrm{ml}$ of $0.125 \%$ levobupivacaine was administered through the catheter, and $300 \mathrm{ml}$ of $0.16 \%$ levobupivacaine mixed with $6 \mathrm{mg}(1.5 \mathrm{ml})$ of indigo carmine was started at $6 \mathrm{ml} / \mathrm{h}$ using a COOPDECH Balloonjector infusion pump (Daiken iki, Osaka, Japan). Indigo carmine was used as a dye to easily identify the leakage of anesthetics. For the use of indigo carmine, we referred to the literature on its use in surgery [10]. The catheter insertion site was covered with a $7 \mathrm{~cm}$ diameter white filter paper to observe local anesthetic leakage. Forty-eight hours after completion of the surgery, the catheter was removed and the degree of staining with indigo carmine was assessed. Postoperative analgesia other than CFNB included celecoxib $200 \mathrm{mg}$ daily at regular doses and acetaminophen up to $2400 \mathrm{mg}$ daily as needed.

\section{Measurement outcome}

The primary outcome was the incidence of anesthetic leakage. Leakage was clearly identified with indigo carmine. We defined no leakage as occurring when the filter paper was not dyed blue at all (Fig. 1A). Secondary outcomes were the incidence at which the $7 \mathrm{~cm}$ diameter filter paper was dyed completely in the cases of leakage, the incidence of catheter malposition, pain scores using the Numerical Rating Scale (NRS) at 48 hours postoperatively, and the number of days until the operated knee could be flexed $120^{\circ}$ postoperatively. Catheter malposition was defined as migration of $>1 \mathrm{~cm}$ or more.

\section{Statistical Analysis}

Fisher's exact test and Wilcoxon rank sum test were conducted to compare each measurement outcome between the CTN and CON groups. Statistical significance was set at $p<0.05$. All statistical analyses were conducted using the IBM SPSS Statistics software version 27 (IBM Corp., Armonk, NY, USA). The sample size was calculated based on a previous study [7] using the Power and Sample Size Program [11], with an alpha error of 0.05 , a detection rate of 0.8 , a leak rate of $80 \%$ for the CTN group, and a leak rate of $30 \%$ for the CON group.

\section{Results}

There were no differences in age, sex, height, weight, body mass index, and anesthetic method (Table 1). Leakages were not present before the start of continuous administration of the local anesthetic in all cases. One patient in the CTN group was excluded from the statistical analysis of measurement outcomes because the catheter was disconnected from the infusion pump before completion of $48 \mathrm{~h}$ of continuous local anesthetic administration. Leakage was significantly larger in the CTN group. In 6 cases in the CON group and 25 cases in the CTN group, a $7 \mathrm{~cm}$ diameter filter paper was dyed completely (Fig. 1B). Five out of 27 leaks in the CTN group and 2 out of 10 leaks in the CON group resulted in a shortened 
catheter insertion length. There was no significant difference in the number of days until the knee joint could be flexed to $120^{\circ}$ after surgery or in the NRS $48 \mathrm{~h}$ after surgery (Table 2 ).

Table 1

Clinical characteristics of patients

\begin{tabular}{|llll|}
\hline Characteristic & CTN group & CON group & P value \\
\hline Number of patients & 30 & 30 & \\
\hline Age, yr & $71 \pm 6$ & $71 \pm 9$ & 0.60 \\
\hline Male / Female, $\mathrm{n}$ & $5 / 25$ & $7 / 23$ & 0.75 \\
\hline Hight, cm & $157 \pm 9$ & $152 \pm 9$ & 0.05 \\
\hline Weight, kg & $69 \pm 15$ & $61 \pm 11$ & 0.11 \\
\hline BMl & $27.1 \pm 4.4$ & $26.5 \pm 4.1$ & 0.66 \\
\hline GA / SA, n & $2 / 28$ & $1 / 29$ & 0.55 \\
\hline $\begin{array}{l}\text { Values are mean } \pm \text { SD or number of patients. Abbreviations: BM/ body mass index, CTN catheter- } \\
\text { through-needle, CON catheter-over-needle, GA general anesthesia, SA spinal anesthesia }\end{array}$ \\
\hline
\end{tabular}

Table 2

Measurement outcomes

\begin{tabular}{|llll|}
\hline & $\begin{array}{l}\text { CTN } \\
\text { group }\end{array}$ & $\begin{array}{l}\text { CON } \\
\text { group }\end{array}$ & $\begin{array}{l}P \\
\text { value }\end{array}$ \\
\hline Number of patients & 29 & 30 & \\
\hline Incidence of leakage, $\mathrm{n}$ & 27 & 10 & 0.001 \\
\hline Incidence at which the filter paper dyed blue completely, $\mathrm{n}$ & 25 & 6 & 0.001 \\
\hline Incidence of catheter malposition, $\mathrm{n}$ & & & 0.20 \\
\hline Pain score at rest after 48 h, median [range] & 5 & 2 & 0.38 \\
\hline $\begin{array}{l}\text { Postoperatively days until the knee could be flexed 120 degrees, } \\
\text { mean } \pm \text { SD }\end{array}$ & $5.9 \pm 4.0$ & $7.3 \pm 4.0$ & 0.13 \\
\hline Abbreviations. CTN catheter-through-needle, CON catheter-over-needle & & $2[0-6]$ \\
\hline
\end{tabular}

\section{Discussion}


This randomized study compared the differences between CTN and CON in regard to the incidence of leakage from the insertion site following CFNB for postoperative analgesia in TKA. The CON group had a significantly lower incidence of leakage from the insertion site than the CTN group, while the other secondary endpoints did not differ between the two groups.

TKA is associated with moderate to severe postoperative pain. Inadequate analgesia can lead to complications such as deep vein thrombosis, pneumonia, and pulmonary embolism, resulting in delayed rehabilitation and prolonged hospital stay [12]. Early achievement of a 120-degree knee flexion position by passive rehabilitation after TKA improves ADL and patient satisfaction $[13,14]$. Various analgesic methods for postoperative pain management in TKA have been developed in clinical settings. Although epidural anesthesia causes side effects such as hypotension, urinary retention, and pruritus, it is known to have better pain reducing effects and lower perioperative opioid consumption than other combinations of analgesic methods for postoperative analgesia in TKA [15]. On the other hand, anticoagulant therapy after lower limb arthroplasty is now widely used to prevent deep venous thrombosis. Therefore, epidural anesthesia is no longer recommended because of the risk of epidural hematoma in patients receiving anticoagulant therapy after surgery [16]. Currently, the combination of femoral nerve block and sciatic nerve block, the method used in this study, is considered the best postoperative analgesic method for TKA [15]. There is no clear guideline on whether femoral nerve block should be administered as a single dose or as a continuous dose during TKA [17]. We believe that postoperative pain is the most severe at the start of rehabilitation, and we prefer to perform CFNB. One of the problems in performing continuous peripheral nerve block is the leakage of local anesthetic from the catheter insertion site. The use of glue at the puncture site $[8,18]$ and tunneling of catheters $[19]$ have been reported to be effective in preventing leakage; however, both of these methods require extra costs and invasive procedures.

In this study, the incidence of leakage was significantly lower in the CON group than in the CTN group. Yu and Nogawa reported that there were no leaks during catheter insertion using a CON needle [7, 9]. Our results showed 10 leaks out of 30 CON cases, and the incidence of leaks was higher when using CON needles than in the previous studies. In our study, leak detection was sensitive due to the addition of dye to the local anesthetic and placing a white filter paper on the puncture site. This may have made it easier to detect leakage compared to previous studies, in which leakage was assessed by visual observation alone. In addition, Nogawa et al. performed a femoral nerve block with a single-use needle and then used a CON needle to place a catheter for CFNB [9]. The use of two different needles may have influenced the leakage from the puncture site.

A $7 \mathrm{~cm}$ diameter filter paper was dyed completely in $60 \%$ (6 patients) of leakage cases in the CON group, and $93 \%$ (25 patients) of leakage cases in the CTN group. This indicates that the degree of leakage was greater in the CTN group than in the CON group. Previous reports have only detected leakage, whereas our evaluation of degree was qualitative, showing the differences in the degree of leakage between CTN and CON using dye. 
There were no differences in the occurrence of catheter malposition, postoperative pain scores, and the number of days to achieve 120 degrees of knee joint flexion in the passive rehabilitation between the CTN and CON groups. Since the incidence and degree of anesthetic leakage from the insertion site were significantly higher in the CTN group, it is possible that the leakage does not affect catheter fixation and postoperative analgesic effects. Leakage may require more frequent changes in dressing or cause patients' clothes to become wet. This may lead to an increase in complaints from nurses and patients.

In contrast to previous reports [7, 9], in this study, opioids were not used for postoperative analgesia. The combination of a single preoperative sciatic nerve block followed by a CFNB and postoperative use of oral nonsteroidal anti-inflammatory drugs (NSAIDs) or acetaminophen could provide effective analgesia after surgery (pain score: median 2 and 1 for CTN and CON groups, respectively). This may indicate that the use of opioids can be avoided for postoperative analgesia in TKA. Since the use of opioids induces vomiting and can cause tolerance and withdrawal symptoms even after a short period of use, our results are also useful to consider the possibility of avoiding the use of postoperative opioids [20].

\section{Study limitations}

Because the nerve block was performed by two practitioners, it is possible that the same technique was not used to place a CFNB catheter in all patients. In addition, we did not measure the time required for the procedure. The technical difficulties in inserting a catheter for CFNB may not be the same in the CTN and $\mathrm{CON}$ groups. There was no significant difference in BMI between the two groups, but we did not compare the thickness of subcutaneous fat at the puncture site, which may have influenced the results. The use of filter paper at the puncture site, which is not commonly used, might have influenced leakage from the puncture site or catheter malposition. The degree of leakage was not assessed quantitatively, but was instead assessed qualitatively, because the $7 \mathrm{~cm}$ filter paper was completely dyed blue in most leakage cases.

\section{Conclusions}

When CFNB was used for analgesia in TKA, the use of CON resulted in less frequent and a lower degree of anesthetic leakage from the insertion site than the use of CTN. Further studies are needed to examine the benefits of reducing leakage by using $\mathrm{CON}$ in patients and healthcare workers.

\section{Abbreviations}

CFNB: Continuous femoral nerve block; CON: Catheter-over-needle; CTN: Catheter-through-needle; NRS: Numerical Rating Scale; NSAID: Nonsteroidal anti-inflammatory drug; TKA: Total knee arthroplasty

\section{Declarations}

\section{Ethics approval and consent to participate}


This study was a prospective, single-center, single blind, randomized controlled trial. The study protocol was approved by the ethics committee of Jikei Medical University Ethics Committee (approval number 27-207(8092)). Written informed consent was obtained from all participants after they understood the concept of this research. The study was implemented in accordance with the principles of the Helsinki Declarations..

\section{Consent for publication}

Consent for publication has been obtained from all participants.

\section{Availability of data and materials}

The datasets used and analyzed during the present study are available from the first author on reasonable request.

\section{Competing interests}

The authors declare that they have no competing interests.

\section{Funding}

Financial support for this study was provided, in part, by departmental funding. The funders had no role in the design of the study, collection and analysis of data, writing manuscript, preparation of the table and figure, or review and revision of manuscript.

\section{Authors' contributions}

$\mathrm{YH}, \mathrm{TH}$ and SO designed the study, $\mathrm{Y}$ Murakami randomized the data, $\mathrm{YH}$, IU and TH analyzed the data, $\mathrm{YH}$ wrote the original draft, $\mathrm{YH}$ and $\mathrm{Y}$ Mio prepared the tables and figures and $\mathrm{YH}, \mathrm{TH}$ and $\mathrm{Y}$ Mio reviewed and edited the tables and figures. All authors revised the manuscript and approved the final version.

\section{Acknowledgements}

We thank Shoichi Uezono MD, Professor and Chair, Department of Anesthesiology, Jikei University School of Medicine for continuous encouragement.

\section{References}


1. Block BM, Liu SS, Rowlingson AJ, Cowan AR, Cowan JA, Wu CL. Efficacy of Postoperative Epidural Analgesia: A Meta-analysis. J Am Med Assoc. 2003;290:2455-63.

2. Vandermeulen EP, Van Aken $\mathrm{H}$, Vermylen J. Anticoagulants and spinal-epidural anesthesia. Anesth Analg. 1994;79:1165-77.

3. Dadure C, Bringuier S, Raux O, Rochette A, Troncin R, Canaud N, et al. Continuous peripheral nerve blocks for postoperative analgesia in children: feasibility and side effects in a cohort study of 339 catheters. Can J Anesth. 2009;56:843-50.

4. Yanovski B, Gaitini L, Volodarski D, Ben-David B. Catastrophic complication of an interscalene catheter for continuous peripheral nerve block analgesia. Anaesthesia. 2012;67:1166-9.

5. Marhofer D, Marhofer P, Triffterer L, Leonhardt M, Weber M, Zeitlinger M. Dislocation rates of perineural catheters: A volunteer study. Br J Anaesth. 2013;111:800-6.

6. Ip VHY, Rockley MC, Tsui BCH. The catheter-over-needle assembly offers greater stability and less leakage compared with the traditional counterpart in continuous interscalene nerve blocks: a randomized patient-blinded study. Can J Anesth. 2013;60:1272-3.

7. Yu B, Hu X, Zou T, He M, Cai G. Effects of Postoperative Continuous Femoral Nerve Block Analgesia with Braun Continuous Peripheral Nerve Block Catheter Set versus Novel Needle-Over-Cannula after Total Knee Arthroplasty. Med Sci Monit. 2015;21:1843-9.

8. Edwards RM, Currigan DA, Bradbeer S, Mitchell C. Does A Catheter over Needle System Reduce Infusate Leak in Continuous Peripheral Nerve Blockade: A Randomised Controlled Trial. Anaesth Intensive Care. 2018;46:468-73.

9. Nogawa R, Maruyama T, Kimoto Y, Yamazaki A, Kawamata T. Comparison of catheter-over-needle and catheter-through-needle on leakage from the catheter insertion site during continuous femoral nerve block. J Anesth. 2018;32:439-42.

10. Osorio JA, Breshears JD, Arnaout O, Simon NG, Hastings-Robinson AM, Aleshi P, et al. Ultrasoundguided percutaneous injection of methylene blue to identify nerve pathology and guide surgery. Neurosurg Focus. 2015;39:1-5.

11. PS: Power and Sample Size Calculation. https://biostat.app.vumc.org/wiki/Main/PowerSampleSize. Access day 15/1/2016

12. Wang F, Zhou Y, Sun J, Yang C. Influences of continuous femoral nerve block on knee function and quality of life in patients following total knee arthroplasty. Int J Clin Exp Med. 2015;8:19120-5.

13. Sakai $\mathrm{N}$, Inoue T, Kunugiza $\mathrm{Y}$, Tomita T, Mashimo T. Continuous femoral versus epidural block for attainment of $120^{\circ}$ knee flexion after total knee arthroplasty: A randomized controlled trial. J Arthroplasty. 2013;28:807-14.

14. Akagi M, Nakamura T, Matsusue Y, Ueo T, Nishijyo K, Ohnishi E. The Bisurface total knee replacement: A unique design for flexion. Four-to-nine-year follow-up study. J Bone Jt Surg - Ser A. 2000;82:1626-33.

15. Terkawi AS, Mavridis D, Sessler DI, Nunemaker MS, Doais KS, Terkawi RS, et al. Pain Management Modalities after Total Knee Arthroplasty: A Network Meta-analysis of 170 Randomized Controlled 
Trials. Anesthesiology. 2017;126:923-37.

16. Horlocker TT, Vandermeuelen E, Kopp SL, Gogarten W, Leffert LR, Benzon HT. Regional Anesthesia in the Patient Receiving Antithrombotic or Thrombolytic Therapy: American Society of Regional Anesthesia and Pain Medicine Evidence-Based Guidelines (Fourth Edition). 2018.

17. Ma T, Liu Q, Zhou L, Yue K, Ding Z, Chen B. Continuous nerve block versus single-shot nerve block for total knee arthroplasty: a meta-analysis from randomized controlled trials. Minerva Anestesiol. 2020;86:205-16.

18. Chalacheewa T, Arnuntasupakul V, Sangkum L, Buachai R, Chanvitayapongs J. Decreasing leakage during continuous femoral nerve catheter fixation using 2-octyl cyanoacrylate glue (Dermabond $\circledast$ ): a randomized controlled trial. BMC Anesthesiol. 2021;21:169.

19. Boezaart AP, de Beer JF, du Toit C, van Rooyen K. A new technique of continuous interscalene nerve block. Can J Anaesth. 1999;46:275-81.

20. Colvin LA, Bull F, Hales TG. Perioperative opioid analgesia-when is enough too much? A review of opioid-induced tolerance and hyperalgesia. Lancet. 2019;393:1558-68.

\section{Figures}



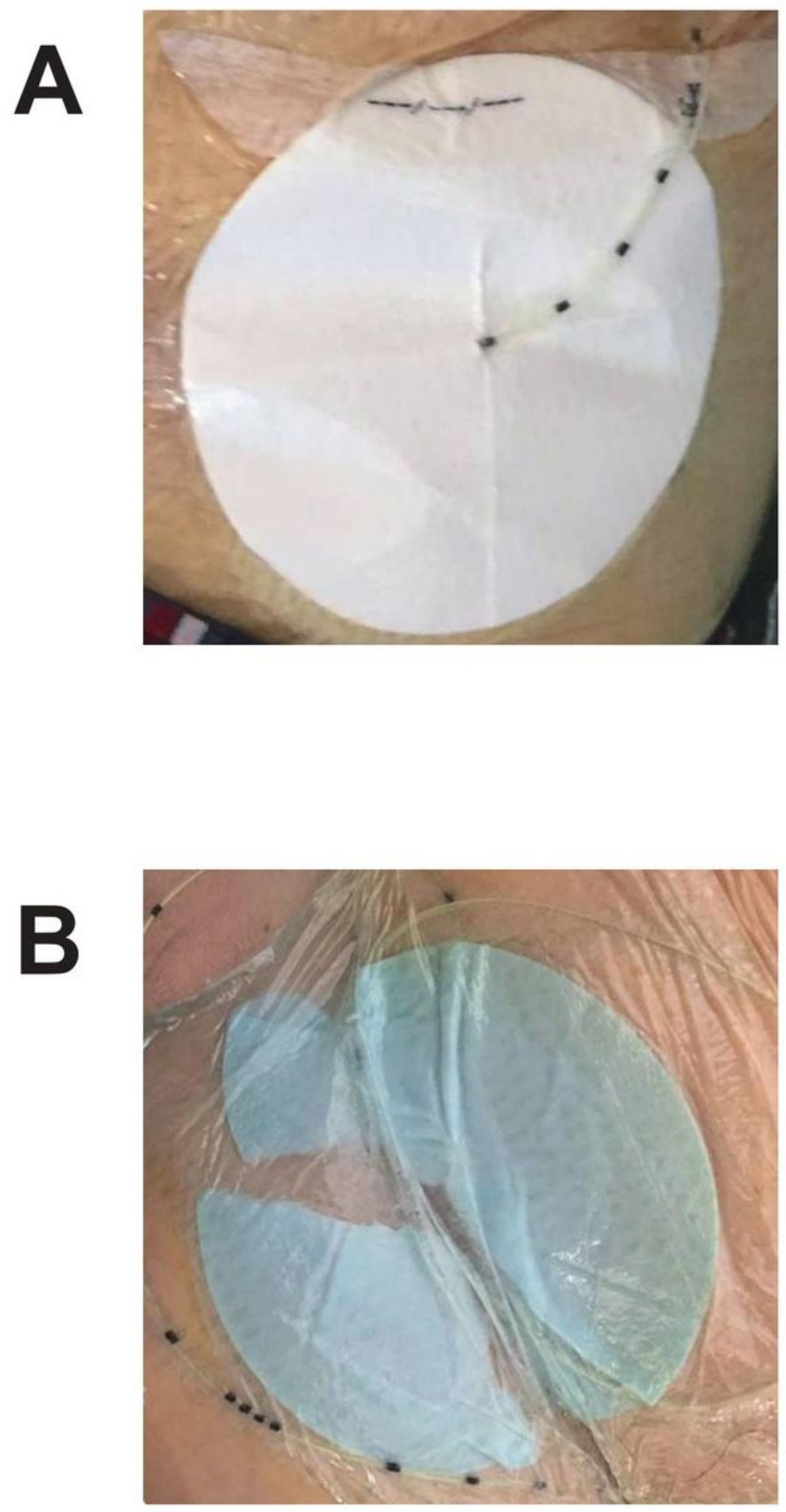

\section{Figure 1}

Catheter insertion site $48 \mathrm{~h}$ after the start of continuous femoral nerve block. A: No leakage. White filter paper was not dyed at all. B: The filter paper was completely dyed blue and the soaked paper was torn. The leakage could be identified easily by adding indigo carmine to local anesthetics. 